\title{
REVIEW
}

\section{Metallic subwavelength-cavity semiconductor nanolasers}

\author{
K. Ding and C. Z. Ning
}

Miniaturization has been an everlasting theme in the development of semiconductor lasers. One important breakthrough in this process in recent years is the use of metal-dielectric composite structures that made truly subwavelength lasers possible. Many different designs of metallic cavity semiconductor nanolasers have been proposed and demonstrated. In this article, we will review some of the most exciting progresses in this newly emerging field. In particular, we will focus on metallic-cavity nanolasers with volume smaller than wavelength cubed under electrical injection with emphasis on high-temperature operation. Such devices will serve as an important component in the future integrated nanophotonic systems due to its ultra-small size.

Light: Science \& Applications (2012) 1, e20; doi:10.1038/lsa.2012.20; published online 20 July 2012

Keywords: Semiconductor Lasers; Nanolasers; Plasmons; Plasmon Polaritons; Metal Cavity

\section{INTRODUCTION}

The field of semiconductor lasers has experienced several paradigm shifts since the first demonstration exactly 50 years ago. ${ }^{1,2}$ Each paradigm shift was made possible by new laser designs, new gain materials or structures made available, and the development of necessary fabrication technologies. Representative examples of these paradigm shifts include the transitions from the original $\mathrm{p}-\mathrm{n}$ junction lasers to double heterostructure lasers, from double heterostructure to quantum well and quantum dot lasers, from Fabry-Perot lasers to distributed feedback lasers and vertical cavity surface emitting lasers (VCSELs), and from VCSELs to photonic crystal lasers, and to various micro-cavity lasers. Each paradigm shift has led to significant improvement in device performance and to new system functionalities. One of the constant themes of these paradigm shifts has been the ability of making ever smaller lasers. Until 5 years ago, laser resonator structures have been almost exclusively dielectric (including semiconductors) based. The sizes of such laser structures are fundamentally limited by the wavelengths involved. As was discussed recently, ${ }^{3}$ the size of dielectric structure lasers is fundamentally limited by two length scales: the half-wavelength and the gain length. Thus, it was apparent that some new wave-guiding or wave-confining mechanisms were needed to shrink the size of lasers significantly further. Metallic structures were the natural choices, since such structures or heavily doped semiconductor layers were used as the wave-confining mechanism for lasers in long-wavelength ranges. ${ }^{4}$ But the situation is different at shorter wavelengths, e.g., near-infrared or visible ranges, due to the significantly larger metal loss. Metal loss compensation by a gain medium was studied in some standard sandwiched waveguide structures at a fixed wavelength. ${ }^{5,6}$ The first laser structure with a semiconductor core as the gain medium, metal shell as a wave-confining mechanism was proposed in $2007^{7,8}$ to significantly reduce the sizes of semiconductor lasers in more than one dimension beyond what is possible with pure dielectric cavities. The systematical analysis included the full frequency dependence of both the real and imaginary parts of the dielectric functions of silver. It was demonstrated that the net modal gain can be achieved by overcoming the metal loss. Any remaining doubt about metallic structures quickly disappeared after the first experimental demonstration of lasing in near infrared wavelengths by Hill et al..$^{9}$ It is interesting to note that micro-cavity lasers and nanolasers prior to 2007 were exclusively based on pure-dielectric cavities, while all the nanolasers after 2007 have metallic structures as essential parts of the cavities. Such new paradigm shift has resulted already in many novel devices with improved performance.

\section{A BRIEF REVIEW OF PROGRESS}

As mentioned above, the first experimental demonstration of metallic cavity nanolaser was reported by Hill et al. ${ }^{9}$ in 2007 . A semiconductorinsulator-metal core-shell structure, similar to what we proposed, ${ }^{7,8}$ but with electrical injection, was fabricated using advanced nanofabrication techniques. InGaAs/InP-based wafer for telecommunication devices was etched into cylindrical pillars of diameter $200-300 \mathrm{~nm}$. Thin SiN layers were then deposited outside before the whole pillar was coated by gold to form a semiconductor-dielectric-metal coreshell structure. Such pillar structures can support hybrid $\mathrm{HE}_{11}$ mode with reasonable $Q$ at the cryogenic temperature. Lasing at the wavelength of $1.4 \mu \mathrm{m}$ was demonstrated on a device with a semiconductor core diameter of $260 \mathrm{~nm}$ at $77 \mathrm{~K}$ under current injection. Remarkably, this result is still the smallest semiconductor laser with electrical injection even until today. The diameter of the circular pillar cavity is only slightly larger than the diffraction limit $(\lambda / 2 n)$. To explore the ultimate limit of size reduction and to confine the optical field below the diffraction limit, later on a metallic-semiconductor structure with a rectangular cross-section was investigated, so that sizes in different directions can be independently reduced. In 2009, we demonstrated lasing in metal-insulator-semiconductor-insulator-metal (MISIM) waveguide at $77 \mathrm{~K}$ with electrical injection. ${ }^{10}$ The thinnest waveguide 
core was only $90 \mathrm{~nm}$, corresponding to a total optical thickness of the devices of about one quarter of the lasing wavelength in active media or one half of the diffraction limit, and supported a transverse magnetic (TM)-like plasmonic gap mode. The interesting aspect of such results is the breaking of the half-wavelength limit, which is assumed typically inevitable in pure dielectric waveguide. Room temperature pulse operation was also achieved on similar devices with thicker waveguide core with a transverse electric (TE)-like mode.

Since then, various types of metallic cavity nanolasers have been demonstrated by many other groups worldwide. Most of these demonstrations were realized under optical pumping. Nezhad et al. studied a circular metallo-dielectric nanocavity laser. ${ }^{11}$ With optimized thickness of dielectric layer, they realized TE mode lasing at room temperature under optical pulse pumping. Later, Lee et al. modified this structure and enabled electrical injection. ${ }^{12}$ An undercut design was introduced to the laser structure to further improve cavity quality. Yu et al. demonstrated lasing from a metal-semiconductormetal sandwich structure called nanopatch laser. ${ }^{13}$ Lasing from both TM and TE mode was observed under optical pumping. Perahia et al. fabricated microdisk lasers with one side covered by metal and showed hybridization of surface-plasmon and dielectric whispering-gallery modes in this structure. ${ }^{14}$ Kwon and his collaborators demonstrated lasing from plasmonic nanodisks. ${ }^{15}$ Recently, lasing from metallic nanoring structures was investigated. ${ }^{16}$ In particular, emission with narrowing linewidth under increasing pumping was observed with a near-unity spontaneous emission factor at $4.5 \mathrm{~K}$. Generally, lasers with electrical injection will be more useful in practical applications than those with optical pumping. Lu et al. demonstrated continuous wave (CW) operation of metallic VCSELs at room temperature. ${ }^{17}$ The dimensions of their devices were, however, over several lasing wavelengths. Significant further miniaturization is needed before such structures can be reduced to subwavelength scale.

The lasing wavelengths of all the examples above are in the region of near infrared where the noble metals (such as silver or gold) serve more as perfect reflecting mirrors. ${ }^{13}$ Other research demonstrated lasing in the visible wavelength which is closer to the metal surface plasmon polariton (SPP) resonance. Deep subwavelength plasmonic lasing ${ }^{18,19}$ was investigated recently. The laser cavities were constructed by placing a CdS nanowire ${ }^{18}$ or nanobelt ${ }^{19}$ on a silver plate covered by thin $\mathrm{MgF}_{2}$ dielectric film. The optical mode was strongly confined in the dielectric film between metal and semiconductor. Lasing around $490 \mathrm{~nm}$ under optical pumping was demonstrated at both cryogenic and room temperature. These results prove that optical gain from semiconductor is capable of compensating for loss in metals in the visible wavelength. Noginov and his collaborators investigated possibility of lasing based on the idea of $\operatorname{spaser}^{20}$ around the wavelength range of $531 \mathrm{~nm}$ using nanoparticles of $44 \mathrm{~nm}$ diameter with a $14 \mathrm{~nm}$-diameter gold core and dye doped silica shell. ${ }^{21}$ The localized surface plasmon oscillation of the gold nanoparticle was sustained by the presence of dye molecules and out-coupled to the photonic mode to be detected. These demonstrations showed important potential for truly subwavelength lasing even close to SPP resonances. However, significant work is still needed to make electrical injection possible at these wavelengths. The wavelength ranges near SPP resonance is interesting in many respects. First, the wavelength compression is maximal at SPP resonance, ${ }^{3,7}$ allowing the smallest lasers to be made although the loss is also maximal. This was considered an essential road-blocker for achieving lasing in these wavelength ranges. A surprising recent study ${ }^{3,22,23}$ has provided new hope. It was shown theoretically that there is an interesting balance of modal loss in the metal and modal gain in the semiconductor gain medium. Such balance is enhanced by a slowing down of energy propagation velocity. Whenever the modal gain can overcome the modal loss, the net modal gain increased dramatically, leading to what is called a giant modal gain. ${ }^{3,22}$ It was shown later that such effect is also related to some peculiar behaviors of confinement factors for metal and semiconductor layers. ${ }^{23}$ The confinement factors can be as large as several orders of magnitude, in stark contrast to the conventional assumption that the confinement factor is always smaller than 1 . Thus, the nearSPP resonance wavelengths contain rich and interesting phenomena that are still not completely explored.

Given the ever increasing attention worldwide and various diverse structures that have been proposed and studied, it is important to compare various structures using the same set of important attributes, or figures of merit to measure the overall progress in this field. The following is a partial list of such quantities:

1. Operation modes: optical pumping $v s$. electrical injection, $\mathrm{CW} v \boldsymbol{s}$. pulse;

2. Operation temperature;

3. Nature of lasing modes (optical or dielectric modes vs. plasmonic modes);

4. Distance of operating wavelengths to the SPP resonances (a certain measure of so-called plasmonicity);

5. Effective volume defined as $V_{\text {phy }} / \lambda^{3}$ where $V_{\text {phy }}$ is the physical volume of the laser cavity and $\lambda$ is the lasing wavelength.

6. Spectral linewidth or value of the $Q$ factor under lasing condition.

Room temperature CW operation under electrical injection is the prerequisite for a nanolaser to be useful for many practical applications. Small effective volume is desired as well to fully explore the advantages of the small size of nanolasers in photonic integration. However, all the subwavelength nanolasers mentioned above are either under optical pumping, ${ }^{11,13-15,18-19,21}$ or CW operation with electrical injection at low temperature ${ }^{9,10,12}$ or room temperature operation but with pulse current injection..$^{10}$ Room temperature CW lasing was demonstrated under electrical injection but with a physical volume of cavity about $12 \lambda^{3} \cdot{ }^{17}$ Only until recently, we have achieved room temperature $\mathrm{CW}$ operation under electrical injection on a metallic cavity laser with cavity volume smaller than $\lambda^{3}{ }^{24}$ Finally, the spectral linewidth under lasing condition is an important quantity. A more appropriate quantity is the $Q$ value under the lasing condition, since this quantity allows a fair comparison of devices that operate at different wavelengths. The comparison of the lasing $Q$ with $Q$ value of the empty cavity can be also useful to compare the relative linewidth improvement from the cavity linewidth.

\section{SEMICONDUCTOR-METALLIC NANOLASERS UNDER ELECTRICAL INJECTION}

We chose to incorporate electrical injection into our device designs from the very beginning because electrical injection is important for many practical applications. Electrical injection is one of the greatest advantages of semiconductor lasers over most of other lasers such as solid-state lasers, where only optical pumping is possible. At the same time, the metal cavity can serve as an electrode and so that no dramatic increase in the complexity of fabrication is required, as in the case of a photonic crystal laser. ${ }^{25}$ Though most demonstrated nanolasers with electrical injection have a cavity with circular cross-section, ${ }^{9,12,17}$ our research more recently has been focused on rectangular cross-section cavities since in this geometry, we can reduce the size of a cavity in each 
a

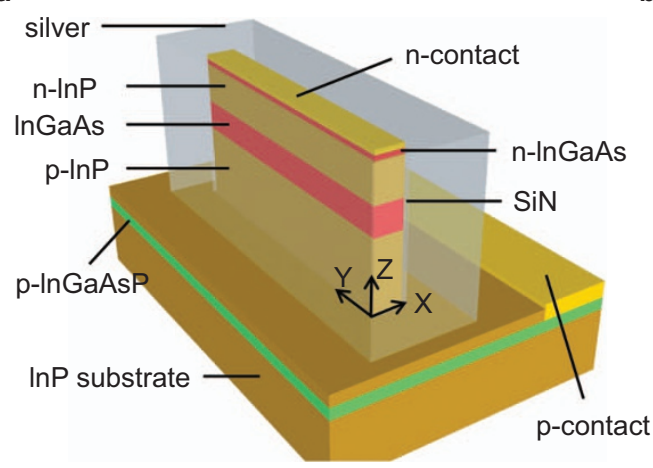

b

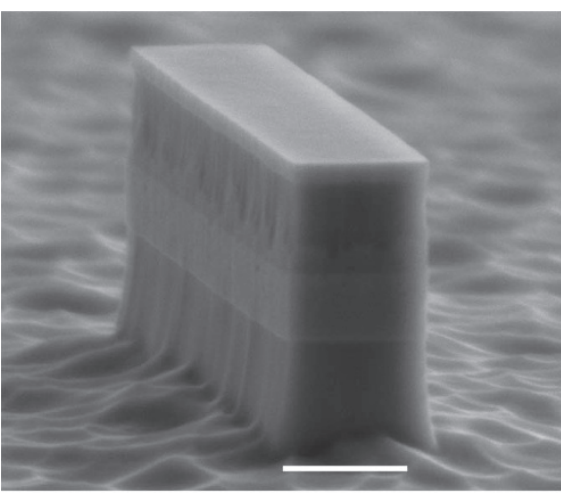

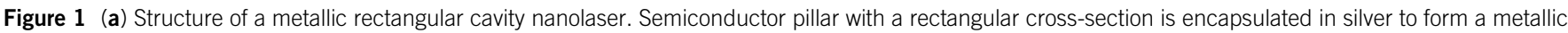
cavity. (b) SEM image of the semiconductor core of the laser structure. Scale bar=500 nm. SEM, scanning electron microscope.

direction independently. Here we will discuss the rectangular cavity nanolasers and our endeavors to realize room temperature CW operation of subwavelength metallic cavity lasers under electrical injection. Several fabrication issues for the electrically driven metallic cavity nanolasers and its possible integration with other photonic components will be discussed as well.

\section{NANOLASERS BASED ON MISIM WAVEGUIDES}

The structure of our semiconductor-metallic nanolasers with rectangular cross-section cavity is shown in Figure 1a. The core of this device consists of an InP/InGaAs/InP rectangular pillar covered with thin silicon nitride insulating layer on all four sides. The InP/InGaAs/ InP layer forms three-layer sandwich waveguide in the vertical direction with a refractive index profile of 3.1 (InP)/3.4 (InGaAs)/ 3.1 (InP), largely confining the optical field in the vertical direction. This pillar is then encapsulated in silver from all four sides as well as from the top, forming a rectangular cavity in the horizontal directions. The $\mathrm{n}$-contact of this device is made through silver and the $\mathrm{p}$ side of the device is connected to an external electric source through the p-type InGaAsP contact layer underneath the cavity. Here the InP/InGaAs/ InP/InGaAsP layer stack was grown via metal-organic chemical vapor deposition on InP substrate. All InGaAs, InGaAsP layers are lattice matched to InP.
A thin rectangular cavity nanolaser is schematically shown in Figure 1a with a large length/width ratio. ${ }^{10,24}$ Such devices have an MISIM cavity in the horizontal plane. Modes of standing waves are formed along the length direction ( $Y$ direction). Depending on the thickness in $X$ direction, either standing wave or gap modes can exist. Part of the mode energy escapes vertically from the InP cladding into the substrate, providing laser emission from the device. Similar waveguides with metal-insulator-metal have been intensively studied both theoretically $^{26-29}$ and experimentally. ${ }^{30,31}$ By inserting InP/InGaAs/ InP heterostructure in the middle, our configuration enables compensation for the metal loss by optical gain in the InGaAs layer. The entire structure can be pumped using electrical injection to achieve optical amplification or lasing.

Two-dimensional (2D) simulation using COMSOL is conducted to study the optical modes propagating in a MISIM waveguide. Mode patterns $(|E|)$ in a $300 \mathrm{~nm}$ wide waveguide are illustrated in Figure 2. One of the modes shown in Figure $2 \mathrm{a}$ is mostly confined in semiconductor core and the other (Figure $2 \mathrm{~b}$ ) is mostly confined in dielectric layer, which is sometimes termed as plasmonic gap mode. ${ }^{28,29}$ In structures with large aspect ratio between $Y$ direction and $X$ direction, the dielectric mode has polarization predominantly along $Z$ direction, while the gap mode is polarized predominantly along $X$ direction. Thus, the two modes resemble TE and TM modes, respectively, propagating along $Y$ direction. Such TE or TM terminology loses meaning

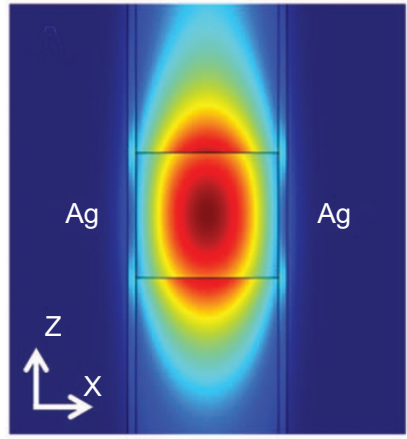

b

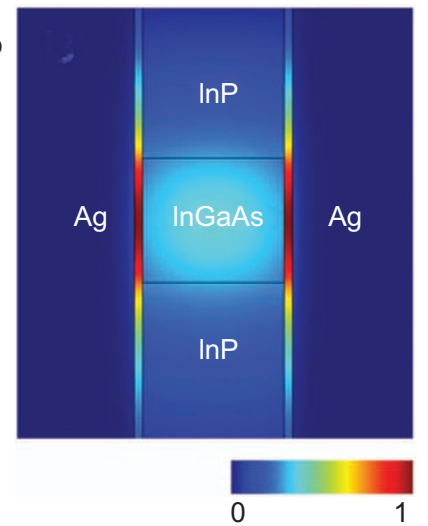

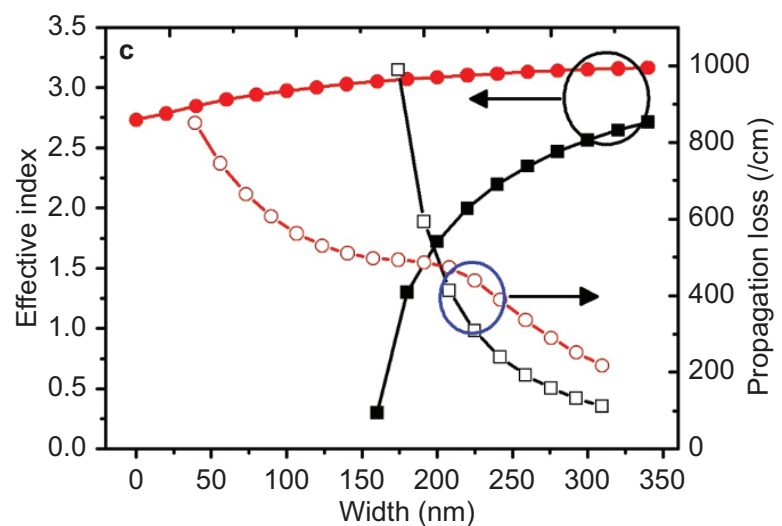

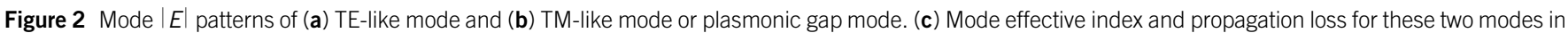

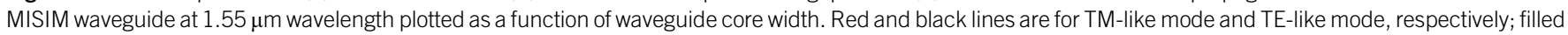

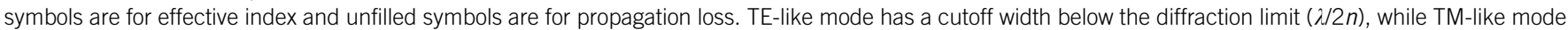
shows no cutoff. MISIM, metal-insulator-semiconductor-insulator-metal; TE, transverse electric; TM, transverse magnetic. 
when the $Y / X$ aspect ratio becomes close to 1 where there is no welldefined propagation direction in a rectangular cavity.

Defining effective modal index as $n_{\mathrm{eff}}=\beta_{\mathrm{w}} / \beta_{0}$ (where $\beta_{\mathrm{w}}$ and $\beta_{0}$ are the wave vectors in MISIM waveguide and free space, respectively), we plot the propagation loss and effective indices of these two modes as a function of semiconductor core width in Figure 2c (assuming $20 \mathrm{~nm}$ SiN layer and wavelength of $1.55 \mu \mathrm{m}$ ). The TE-like mode shows a cutoff around $200 \mathrm{~nm}$ (roughly $\lambda / 2 n$, or the diffraction limit) as indicated by a dramatically decreased index and increased modal loss, while the plasmonic gap mode exists even when the width of the waveguide core approaches zero. Although the index decreases slightly for the TM-like mode, the loss increases significantly with the decrease of the core width. Theoretically, such a MISIM waveguide can be made arbitrarily thin to support the plasmonic gap mode. Based on this idea, we have demonstrated lasing around $1.35 \mu \mathrm{m}$ in a MISIM waveguide cavity with $90 \mathrm{~nm}$ semiconductor core width and $6 \mu \mathrm{m}$ length, propagating a $\mathrm{TM}_{0}$ plasmonic gap mode at $10 \mathrm{~K}$ under DC current injection. ${ }^{10}$ Considering the $15-\mathrm{nm}$ SiN layer and several nanometers of penetration depth into silver, the total optical width of this waveguide is around $380 \mathrm{~nm}$, which is about a quarter of the lasing wavelength in the vacuum, far below the diffraction limit of the half of a wavelength. To our best knowledge, this is the first demonstration of any semiconductor laser at deep subwavelength scale below the diffraction limit.

As shown in Figure 2c, when the width of the waveguide exceeds $200 \mathrm{~nm}$, the TE-like mode, confined mostly in semiconductor core, has a low loss and large gain confinement factor (Figure $2 \mathrm{a}$ and $2 \mathrm{~b}$ ), thus becoming the dominant mode. In general, wider devices that support a TE-like mode can operate at higher temperature than the thin plasmonic gap mode devices. We have achieved pulse lasing at room temperature on a device of $310 \mathrm{~nm}$ in width and $6 \mu \mathrm{m}$ in length. ${ }^{10}$ The length of the wide TE-like mode device can be significantly shortened while maintaining a reasonable cavity $Q$ factor. In terms of total cavity volume, the TE-like mode device can be even smaller. We have observed $\mathrm{CW}$ lasing at $100 \mathrm{~K}$ on a device with $400 \mathrm{~nm}$ in width and $900 \mathrm{~nm}$ in length. The total volume of the device cavity is only $0.19 \lambda^{3}$, smaller than the plasmonic gap mode devices that we have reported in Ref. 10. Figure 3 shows the spectra of this device at different currents at $100 \mathrm{~K}$ and the light-current curve on a log-log scale. The lasing wavelength of this device is $1454 \mathrm{~nm}$, far from the

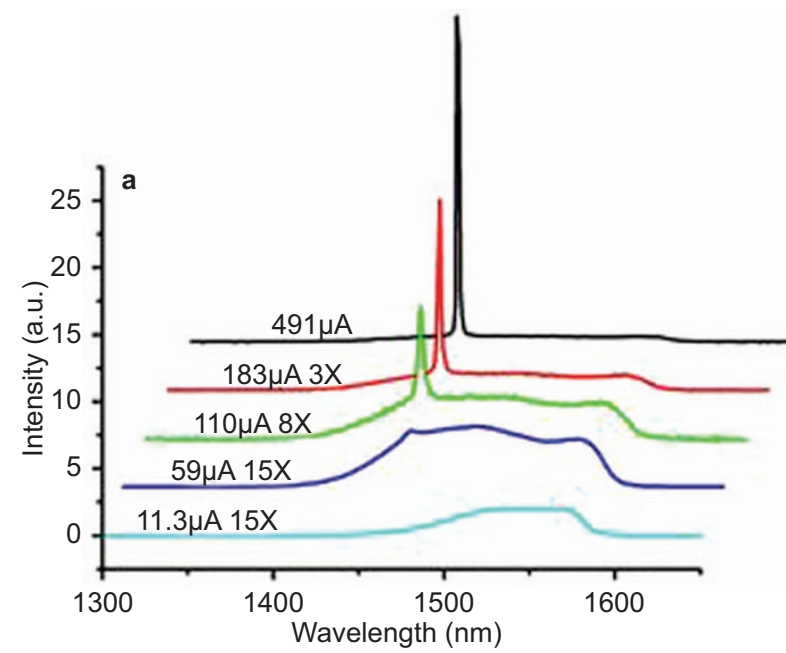

band-edge emission wavelength of InGaAs material at $100 \mathrm{~K}$. Given that the cavity length of this device is only $900 \mathrm{~nm}$, the mode spacing is very large in this device and there is likely only one mode within material gain bandwidth. Due to the mismatch between the gain maximum and the modal wavelength, such lasers can only lase at a much higher injection level. Therefore, an important strategy to reduce threshold current for such small lasers is to match the cavity resonance and material gain peak, which can be achieved by careful design of the cavity size to tune its resonance or optimize gain material composition.

Due to the smaller physical volume, an important property of such deep subwavelength cavity lasers is the enhanced spontaneous emission coupled to the lasing mode, also known as the Purcell effect. A large Purcell factor $F=29.27$ is estimated for this device, corresponding to an effective mode volume of $5.4\left(\lambda / 2 n_{\text {eff }}\right){ }^{3}$ The influence of the Purcell effect can be demonstrated in the following rate equations. ${ }^{32,33}$

$$
\begin{aligned}
& \frac{\mathrm{d} N}{\mathrm{~d} t}=\frac{I}{q V_{\mathrm{a}}}-v_{\mathrm{g}} G S-\frac{1-\beta_{0}}{\tau_{\mathrm{sp}}} N-\frac{F \beta_{0}}{\tau_{\mathrm{sp}}} N-\frac{v_{\mathrm{s}} S_{\mathrm{a}}}{V_{\mathrm{a}}} N-\frac{N}{\tau_{\mathrm{a}}} \\
& \frac{\mathrm{d} S}{\mathrm{~d} t}=\Gamma v_{\mathrm{g}} G S+\frac{\Gamma F \beta_{0}}{\tau_{\mathrm{sp}}} N-\frac{S}{\tau_{\mathrm{ph}}}
\end{aligned}
$$

The definitions of the parameters are listed in Table 1.

Through rate equation calculation, $\beta_{0}$ is fitted to be 0.004 . Spontaneous emission factor defined as ratio of spontaneous emission coupled to the lasing mode to the total emission, or $\beta=F \beta_{0} /$ $\left(1-\beta_{0}+F \beta_{0}\right)$, is calculated to be 0.105 . This large spontaneous emission factor is two to three orders larger than that in conventional ridge waveguide lasers, ${ }^{34,35}$ one order larger than in typical VCSELs ${ }^{36}$ and similar to the reported values for microdisk lasers and photonic crystal lasers. ${ }^{37,38}$

\section{ROOM TEMPERATURE, CW OPERATION UNDER ELECTRICAL INJECTION OF A SUBWAVELENGTH-CAVITY LASER}

The realization of CW operation under electrical injection at room temperature is an important step towards practical applications of metallic cavity nanolasers. Such demonstration will also have a major impact on the research in plasmonic systems and metamaterials where composites of metallic and semiconductor structures are required. Despite the intensive worldwide pursuit, room temperature operation

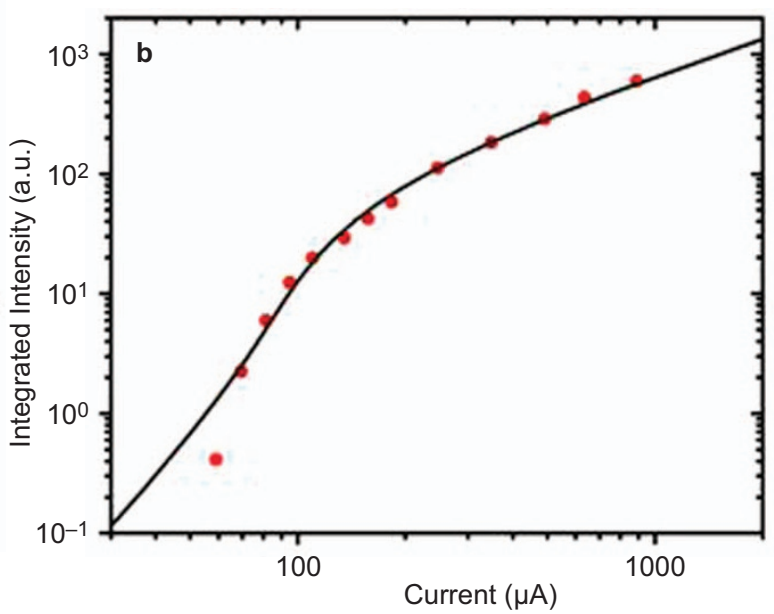

Figure 3 (a) Spectra of a device with volume of $400 \mathrm{~nm} \times 900 \mathrm{~nm} \times 1.55 \mu \mathrm{m}=0.19 \lambda^{3}$ at $100 \mathrm{~K}$. (b) $L-/$ curve in log-log scale from experiment (dots) and rate equation calculation (solid line). 
Table 1 Definitions of parameters in rate equations

\begin{tabular}{ll}
\hline Parameter & \\
\hline$N$ & Carrier density \\
$S$ & Photon density \\
$v_{\mathrm{g}}$ & Group velocity \\
$\tau_{\mathrm{sp}}$ & Spontaneous emission life time into large 3D space \\
$V_{\mathrm{a}}$ & Active region volume \\
$V_{\mathrm{s}}$ & Surface recombination velocity \\
$S_{\mathrm{a}}$ & Active region surface area exposed to SiN layer \\
$\tau_{\mathrm{au}}$ & Auger recombination life time \\
$\Gamma$ & Confinement factor \\
$\mathrm{G}$ & Material gain \\
$\tau_{\mathrm{ph}}$ & Photon life time \\
$\beta_{0}$ & Spontaneous emission factor without the Purcell enhancement \\
$F$ & Purcell factor \\
\hline
\end{tabular}

Abbreviation: 3D, three-dimensional.

of subwavelength metallic cavity lasers have been limited to optical pumping or pulse electrical injection due to the significant heating effect associated with CW operation under electrical injection.

One major loss mechanism in metallic cavity lasers comes from the overlap of the optical field with the lossy metal which is directly related to the surface area of the cavity. Given the large surface/volume ratio of these subwavelength cavity devices, the carrier life time in the active region will be significantly reduced by the surface recombination. These two issues are both related to the surface/volume ratio of the metallic cavity. Therefore, one obvious strategy to improve the cavity quality of our rectangular device optically and electrically is to reduce the aspect ratio, so the structures are more square-like with less surface area at the same total volume. Figure 4 shows the cavity quality factors from finite-difference time-domain simulation for $2 \mathrm{D}$ rectangular silver-SiN-InGaAs cavities with different length/width ratios but the same area of $1.9 \mu \mathrm{m}^{2}$ which is about $0.8 \lambda^{2}(\lambda=1.55 \mu \mathrm{m})$. Clearly with aspect ratio of 3-5, the cavity has the highest $Q$. In this simulation, the TE mode with electric field in $Z$ axis is simulated. In TE mode, the electric field is parallel to metal surface and has least interaction with the lossy metal, thus leading to less metal loss and higher $Q$.

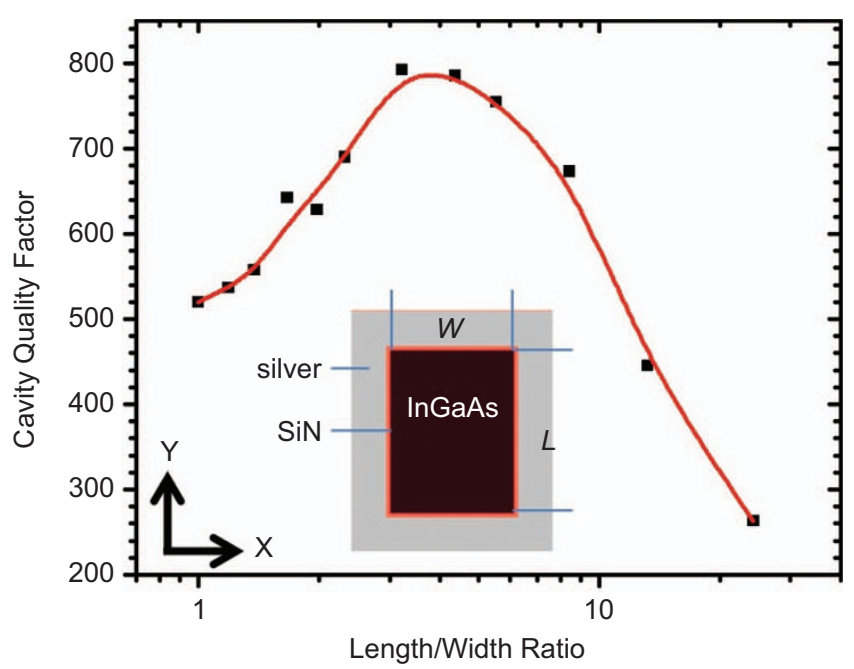

Figure 4 Cavity quality factors of 2D rectangular silver-SiN-InGaAs cavities of different length/width ratios but same total area of $0.8 \lambda^{2}$. Inset is the simulation geometry. 2D, two-dimensional.
Although this 2D simulation does not necessarily represent exactly the real three-dimensional situation, it does show the improvement of cavity quality factor in a rectangular cavity with proper aspect ratio compared to the narrow waveguide with a large aspect ratio. We fabricated a series of devices with rectangular cross-section having different aspect ratios. Improvement CW operation temperature is observed as expected with decreasing length/width ratio. One device with dimension of $1.1 \mu \mathrm{m}$ (width) $\times 2.15 \mu \mathrm{m}$ (length) $\times 1.55 \mu \mathrm{m}$ (height $)=0.96 \lambda^{3}(\lambda=1563.4 \mathrm{~nm})$ can lase in CW mode at a high temperature of $260 \mathrm{~K},{ }^{39}$ which can be achieved by thermoelectric cooling. Later, we reported the first demonstration of room temperature CW operation on subwavelength metallic cavity lasers. ${ }^{24}$ The total device volume is $0.95 \lambda^{3}$ and the lasing linewidth is $3 \mathrm{~nm}$ (Figure 5). Though the broad linewidth and smooth threshold transition in this demonstration can be attributed to the severe self-heating at room temperature under CW electrical injection, it did raise questions about the capability of such subwavelength metallic cavity lasers operating at room temperature with CW performance comparable to conventional semiconductor lasers. In addition, we occasionally observed good lasing from thin waveguide type devices at high temperatures around $250 \mathrm{~K}$, which led one to believe that significantly enhanced performance on rectangular cavity devices with proper shape is definitely possible. Recently, with improved fabrication process and device design, we achieved unambiguous room temperature CW lasing from a rectangular cavity device with a total volume of $0.67 \lambda^{3}{ }^{40}$ This device showed a clear turn-on threshold and narrow linewidth down to $0.5 \mathrm{~nm}$. Such demonstration proves that electrically driven subwavelength metallic cavity lasers can operate far above threshold under CW operation at room temperature and show lasing characteristics similar to conventional semiconductor lasers. The preliminary results have been reported at 2012 CLEO and details will be published elsewhere. ${ }^{40}$ This result is comparable to or better than other best demonstrations of semiconductor nanolasers at room temperature (Table 2).

\section{DEVICE FABRICATION ISSUES}

Theoretical studies ${ }^{3,41-45}$ have predicted many appealing features of metallic nanolasers such as small volume, high density integration and good thermal dissipation. Such small lasers potentially can operate at high modulation speed. ${ }^{46-48}$ Many such features have not been realized due to the fabrication related issues. This is especially so, since the effects of imperfection in fabrication become much more severe on the nanoscale. Very often, the performance of fabricated devices falls significantly short of theoretical expectation. Before the eventual realization of theoretical predictions, several manufacture-related issues need to be addressed properly. The sidewalls of the semiconductor pillar need to be smooth and vertical. Simulation shows that even a $2^{\circ}$ inclination of the sidewalls can lead to a significant drop of cavity $Q$ factor. ${ }^{49}$ The quality of the dielectric layer is also very critical. It needs to be strong enough so that it would not break down when high voltage is applied. Given the high surface/volume ratio, strong surface recombination will be detrimental to the nanocavity devices. ${ }^{50}$ Ideally, the dielectric layer should effectively passivate the surface of semiconductor to reduce the surface recombination. The design of dielectric layer thickness is also a very delicate issue. Though simulations show that a thick dielectric layer $(100-200 \mathrm{~nm})^{41}$ will spatially separate the optical field and lossy metal, thus improving the cavity quality, we have observed in our experiments that devices with a thicker SiN layer tend to have a shorter lifetime. Such reliability issues are likely related to the stress accumulated in thick SiN layer given the significant difference between the thermal expansion coefficients of $\mathrm{SiN}$ and $\mathrm{InP} /$ 


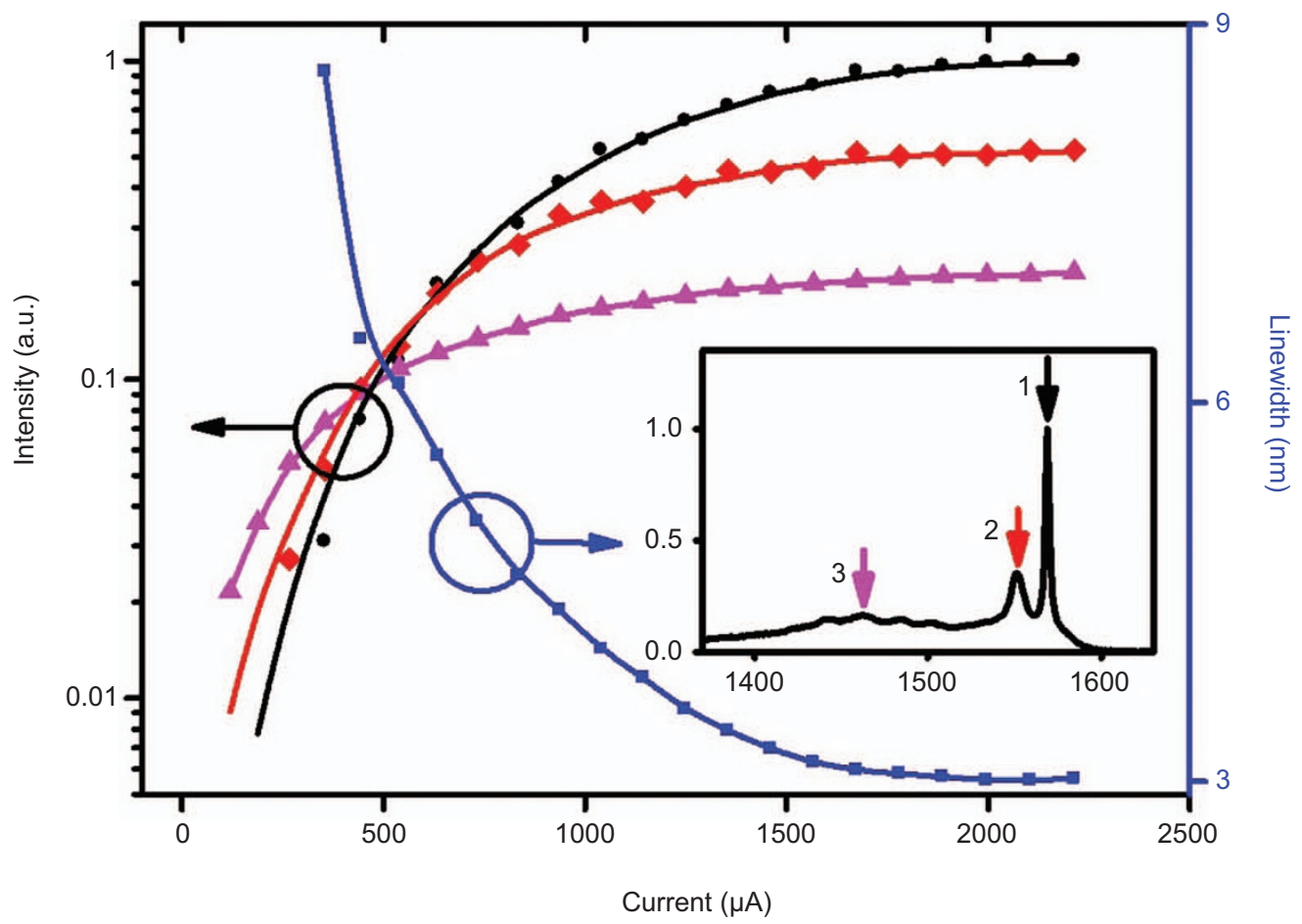

Figure 5 Light intensity and linewidth of a device with total volume of $1.1 \mu \mathrm{m}$ (width) $\times 2.15 \mu \mathrm{m}$ (length) $\times 1.55 \mu \mathrm{m}$ (height) $=0.95 \lambda^{3}$ at room temperature of $293 \mathrm{~K}$ under dc current injection. Inset: spectrum with arrows and numbers indicating positions where light intensity is taken: lasing mode ( circles, 1), non-lasing mode ( diamonds, 2) and spontaneous emission at $1460 \mathrm{~nm}$ ( $\boldsymbol{\Delta}$ triangles, 3). The final lasing linewidth is $3 \mathrm{~nm}$. A smooth threshold current is estimated $\sim 1000 \mu \mathrm{A} .{ }^{24} \mathrm{dc}$, direct current.

InGaAs. This practical issue needs to be carefully considered during device design and fabrication.

Recently, due to the intensive studies in metallic plasmonic systems, the optical qualities of noble metals also attracted special attention. Different deposition techniques for noble metals, such as silver and gold, have been investigated, aiming to obtain smooth and low loss metal layers. It was found that a thin germanium wetting layer $(\sim 1 \mathrm{~nm})$ before silver deposition can significantly reduce the surface roughness and sheet resistance of a thin silver film. ${ }^{51}$ Proper annealing process will reduce the loss in silver films as well. ${ }^{52}$ Atomic layer deposition of silver is under investigation ${ }^{53}$ to meet the requirement for film uniformity and thickness control in certain plasmonic devices. ${ }^{54}$ Another interesting approach ${ }^{55}$ that has attracted the attention of the community is the replacement of metals by highly doped semiconductors, as was done for long wavelengths. ${ }^{4,56}$ A recent study ${ }^{55}$ shows that highly doped InAs in InAs/GaSb or InAs/AlSb heterostructures can be a good metallic material for near-infrared wavelengths, with much reduced metal loss than Ag or Au. The advantage of such all-semiconductor-based approach is that the entire structure can be fabricated using epitaxial growth, without the need of thermal deposition of metals.

\section{NANOLASER INTEGRATION WITH SILICON WAVEGUIDE}

Due to the small output facet and strong wave guiding in a metallic nanocavity laser, far-field divergence is an important issue to address for practical applications of such small lasers. Far-field coupling would become impractical due to the increased beam divergence. Direct integration of nanolasers with waveguides becomes a necessity. Silicon-based waveguides, among many choices, become attractive. Silicon-based optoelectronics integration offers the promise of low cost and compact package solution for optical communications and interconnects. ${ }^{57,58}$ An electrically pumped coherent light source on silicon is a key element needed for integrated photonic circuits on silicon. Considering its small volume, the nanolaser device is one of

Table 2 A summary of recent room temperature subwavelength semiconductor nanolasers

\begin{tabular}{|c|c|c|c|c|c|}
\hline References & Cavity $Q$ & Lasing $Q$ & Linewidth/nm & Wavelength/nm & Operation mode \\
\hline Ding et $a .^{24}$ & $\sim 240$ & 523 & 3 & 1568 & CW, electrical \\
\hline Ding et al. ${ }^{40}$ & 235 & 3182 & 0.5 & 1591 & CW, electrical \\
\hline Hill et al. ${ }^{10}$ & 340 & 3000 & 0.5 & 1500 & Pulse, electrical \\
\hline Nezhad et al. ${ }^{11}$ & Not reported & 2860 & 0.5 & 1430 & Pulse, optical \\
\hline Perahia et al. ${ }^{14}$ & Not reported & 6500 & $\sim 0.2$ & 1300 & Pulse, optical \\
\hline Ma et al. ${ }^{19}$ & 97 & 495 & $\sim 1$ & $\sim 495$ & Pulse, optical \\
\hline Chen et al. ${ }^{50}$ & 206 & 317 & $\sim 3$ & $\sim 950$ & Pulse, optical \\
\hline Khajavikhan et al. ${ }^{16}$ & 53 & $\sim 215$ & $\sim 7$ & $\sim 1500$ & CW, optical \\
\hline
\end{tabular}

Abbreviation: CW, continuous wave. 

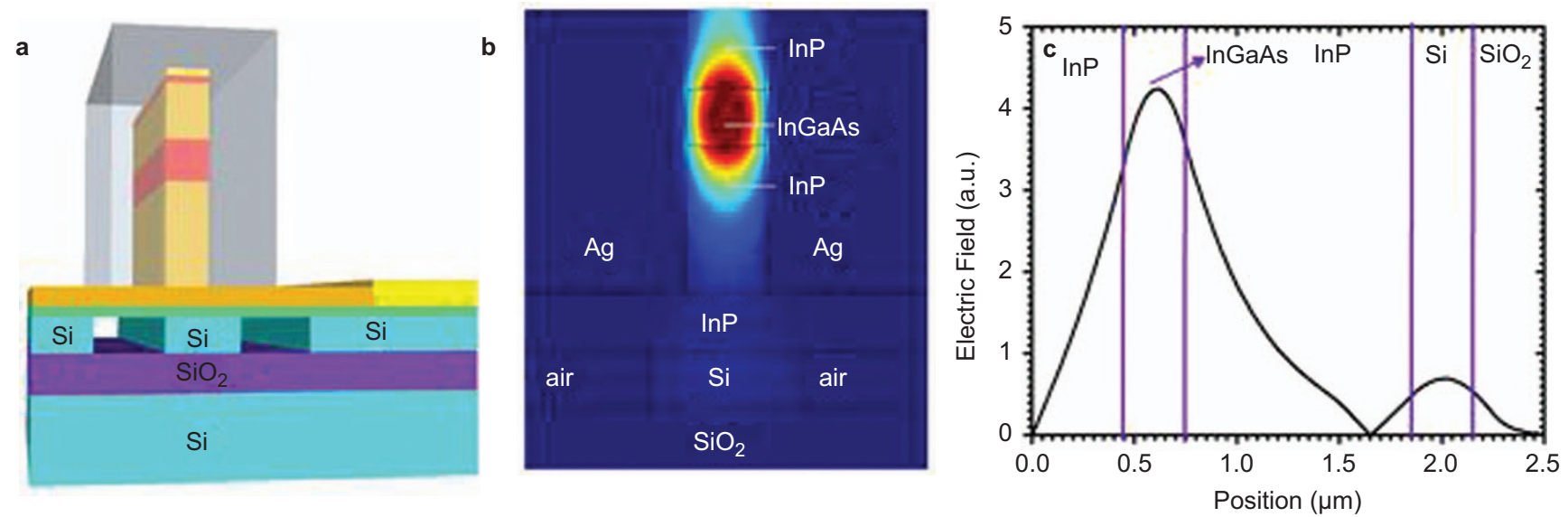

Figure 6 (a) Structure of a nanolaser/silicon waveguide integration based on evanescent coupling. (b) $|E|$ from 2D simulation for MISIM waveguide on silicon waveguide. (c) Electric field distribution in the vertical direction along the center of the waveguide, showing sufficient evanescent coupling between a $400 \mathrm{~nm}$ wide nanolaser waveguide and an $800 \mathrm{~nm}$ wide and $300 \mathrm{~nm}$ thick silicon waveguide. 2D, two-dimensional.

the promising candidates to fulfill the role of a light source on silicon to build high-density integrated photonic circuits on silicon. A possible design is to integrate the nanolaser device directly on top of the silicon waveguide on a silicon-on-insulator substrate. Our design with backside coupling shows an advantage for such coupling. Figure $6 \mathrm{a}$ shows an example of such integration of our subwavelength laser on top of a silicon waveguide. Through 2D simulation (Figure $6 \mathrm{~b}$ and $6 \mathrm{c}$ ), we found an evanescent coupling can be efficient if the effective indices of the two waveguides match. Kim et al. proposed a similar evanescent coupling scheme ${ }^{59}$ to integrate a metallic cavity nanolaser with a silicon-on-insulator waveguide. Simulation shows that a high coupling efficiency can be achieved between the metallic nanocavity and the silicon-on-insulator waveguide underneath. Another end-fire coupling scheme for metallic nanopatch laser is also studies by Ding et al ${ }^{60}$ Serious experimental efforts are still needed before such an integrated system becomes a reality.

\section{CONCLUSION}

The last few years have witnessed a dramatic size reduction of semiconductor lasers since the first demonstration of metallic cavity nanolasers $^{9}$ and the original proposal. ${ }^{7,8}$ Unlike nanowire lasers or microdisk lasers, the light confinement through metallic structures can overcome the fundamental size limits of the pure dielectric lasers imposed by the wavelength involved. ${ }^{3}$ Many groups have demonstrated various metallic cavity nanolasers with different designs. Now the size of the smallest metallic cavity nanolaser is already comparable to state-of-the-art electronic transistors in at least one dimension. Many interesting physical phenomena on the nanoscale were also studied on the platform of metallic cavity nanolasers. In this review, we briefly reviewed our work on metallic cavity nanolasers, focusing on size reduction and high-temperature operation. Theoretically, the optical gain in semiconductor is more than enough to overcome the loss in deep subwavelength metallic cavity even at room temperature, so that good performances comparable to conventional semiconductor lasers are possible in ultra-small nanolasers. Currently, imperfection of nanofabrication technology is one of the major bottlenecks in the development of metallic cavity nanolasers. We believe that, with the evolution of fabrication technology addressing the practical problems, such as surface passivation and material deposition, all the interesting theoretical predictions about metallic cavity nanolasers will be realized eventually. New physics phenomena at the nanoscale are expected. More importantly, applications of metallic cavity nanolasers, especially its integration with other photonic components, will greatly impact the future nanophotonics systems.

\section{ACKNOWLEDGMENTS}

Research reported in this article in the authors group was supported by the Defense Advanced Research Project Agency program Nanoscale Architectures of Coherent Hyper-Optical Sources (grant no. W911-NF07-1-0314) and by the Air Force Office of Scientific Research (grant no. FA9550-10-1-0444, Gernot Pomrenke). We thank Martin Hill for his collaboration over the last few years.

1 Hall RN, Fenner GE, Kingsley JD, Soltys TJ, Carlson RO. Coherent light emission from GaAs junctions. Phys Rev Lett 1962; 9: 366-368.

2 Nathan MI, Dumke WP, Burns G, Dill FH, Lasher G. Stimulated emission of radiation from GaAs p-n junctions. Appl Phys Lett 1962; 1: 62-64.

3 Ning CZ. Semiconductor nanolasers. Phys Stat Solidi B 2010; 247: 774-788.

4 Sirtori C, Gmachl C, Capasso F, Faist J, Sivco DL et al. Long-wavelength $(\lambda \approx 8$ $11.5 \mu \mathrm{m}$ ) semiconductor lasers with waveguides based on surface plasmons, Opt Lett 1998; 23: 1366-1368.

5 Nezhad MP, Tetz K, Fainman Y. Gain assisted propagation of surface plasmon polaritons on planar metallic waveguides. Opt Express 2004; 12: 4072-4079.

6 Maier SA. Gain-assisted propagation of electromagnetic energy in subwavelength surface plasmon polariton gap waveguides. Opt Commun 2006; 258: 295-299.

7 Maslov AV, Ning CZ. Size reduction of a semiconductor nanowire laser using metal coating. Proc SPIE 2007; 6468: 646801-646807.

8 Maslov AV, Ning CZ. Metal-encased semiconductor nanowires as waveguides for ultrasmall lasers. In: Proceedings of the Conference on Laser and Electro-Optics 2007; 6-11 May 2007; Baltimore, MD, USA. OSA: Washington, DC, USA, 2007, Paper JWA121.

9 Hill MT, Oei YS, Smalbrugge B, Zhu YC, Veries TD et al. Lasing in mtellic-coated nanocavities. Nat Photonics 2007; 1: 589-594.

10 Hill MT, Marell M, Leong ES, Smalbrugge B, Zhu YC et al. Lasing in metal-insulatormetal sub-wavelength plasmonic waveguides. Opt Express 2009; 17: $11107-$ 11112.

11 Nezhad MP, Simic A, Bondarenko O, Slutsky B, Mizrahi A et al. Room-temperature subwavelength metallo-dielectric lasers. Nat Photonics 2010; 4: 395-399.

12 Lee JH, Khajavikhan M, Simic A, Gu Q, Bondarenko Q et al. Electrically pumped sub-wavelength metallo-dielectric pedestal pillar lasers. Opt Express 2011; 19: 21524-21531.

13 Yu K, Lakhani A, Wu MC. Subwavelength metal-optic semiconductor nanopatch lasers. Opt Express 2010; 18: 8790-8799.

14 Perahia R, Mayer Alegre TP, Safavi-Naeini AH, Painter O. Suface-plasmon mode hybridization in subwavelength microdisk lasers. Appl Phys Lett 2009; 95: 201114-201116.

15 Kwon SH, Kang JH, Seassal C, Kim SK, Regreny P et al. Subwavelength plasmonic lasing from a semiconductor nanodisk with silver nanopan cavity. Nano Lett2010; 10: 3679-3683.

16 Khajavikhan M, Simic A, Katz M, Lee JH, Slutsky B et al. Thresholdless nanoscale coaxial lasers. Nature 2012; 482: 204-207. 
17 Lu CY, Chang SW, Chuang SL, Germann TD, Bimberg D. Metal-cavity surface-emitting microlaser at room temperature. App/ Phys Lett 2010; 96: 251101-251103.

18 Oulton RF, Sorger VJ, Zentgraf T, Ma RM, Gladden C et al. Plasmon lasers at deep subwavelength scale. Nature 2009; 461: 629-632.

19 Ma RM, Oulton RF, Sorger VJ, Bartal G, Zhang X. Room-temperature sub-diffractionlimited plasmon laser by total internal reflection. Nat Mater 2011; 10: 110-113.

20 Bergman DJ, Stockman MI. Surface plasmon amplification by stimulated emission of radiation: quantum generation of coherent surface plasmon in nanosystems. Phys Rev Lett 2003; 90: 027402-027405.

21 Noginov MA, Zhu G, Belgrave AM, Bakker R, Shalaev VM et al. Demonstration of a spacer-based nanolaser. Nature 2009; 460: 1110-1112.

22 Li DB, Ning CZ. Giant modal gain, amplified surface plasmon-polariton propagation, and slowing down of energy velocity in a metal-semiconductor-metal structure. Phys Rev B 2009; 80: 153304-153307.

23 Li DB, Ning CZ. Peculiar features of confinement factors in a metal-semiconductor waveguide, App/ Phys Lett 2010; 96: 181109-181111.

24 Ding K, Liu ZC, Yin LJ, Hill MT, Marell MJ et al. Room-temperature continuous wave lasing in deep-subwavelength metallic cavities under electrical injection. Phys Rev $B$ 2012; 85: 041301-041305.

25 Park HG, Kim SH, Kwon SH, Ju YG, Yang JK et al. Electrically driven single-cell photonic crystal laser. Science 2004; 305: 1444-1447.

26 Zia R, Selker MD, Catrysse PB, Brongersma ML. Geometries and materials for subwavelength surface plasmon modes. J Opt Soc Am A 2004; 21: 2442-2446.

27 Kusunoki F, Yotsuya T, Takahara J, Kobayashi T. Propagation properties of guided waves in index-guided two-dimensional optical waveguides. Appl Phys Lett 2005; 86: 211101-211103.

28 Tanaka K, Tanaka M. Simulations of nanometric optical circuits based on surface plasmon polariton gap waveguide. Appl Phys Lett 2003; 82: 1158-1160.

29 Feng NN, Brongersma ML, Negro LD. Metal-dielectric slot-waveguide structures for the propagation of surface plasmon polaritons at $1.55 \mu \mathrm{m}$. IEEE J Quantum Electron 2007; 43: 479-485.

30 Miyazaki HT, Kurokawa Y. Squeezing visible light waves into a 3-nm-thick and 55-nmlong plasmon cavity. Phys Rev Lett 2006; 96: 097401-097404.

31 Kaminow IP, Mammel WL, Weber HP. Metal-clad optical waveguides: analytical and experimental study. Appl Opt 1974; 13: 396-405.

32 Coldren LA, Corzine SW. Diode lasers and photonic integrated circuits. New York: Wiley, 1995.

33 Baba T, Sano D. Low-threshold lasing and Purcell effect in microdisk lasers at room temperature. IEEE J Sel Top Quantum Electron 2003; 9: 1340-1346.

34 Petermann K. Calculated spontaneous emission factor for double-heterostructure injection lasers with gain-induced waveguiding. IEEE J Quantum Electron 1979; 15: 566-570.

35 Goodwin JC, Garside BK. Measurement of spontaneous emission factor for injection lasers. IEEE J Quantum Electron 1982; 18: 1264-1271.

36 Shin JH, Ju YG, Shin HE, Lee YH. Spontaneous emission factor of oxidized verticalcavity surface-emitting lasers from the measured below-threshold cavity loss. App/ Phys Lett 1997; 70: 2344-2346.

37 Fujita M, Ushigome R, Baba T. Large spontaneous emission factor of 0.1 in a microdisk injection laser. IEEE Photon Technol Lett 2001; 13: 403-405.

38 Ryu HY, Notomi M, Kuramoti E, Segawa T. Large spontaneous emission factor $(>0.1)$ in the photonic crystal monopole-mode laser. App/ Phys Lett 2004; 84: 10671069.

39 Ding K, Liu ZC, Yin LJ, Wang H, Liu RB et al. Electrical injection, continuous wave operation of subwavelength-metallic cavity lasers at 260 K. App/ Phys Lett 2011; 98 : 231108-231110.
40 Ding K, Hill MT, Liu ZC, Yin LJ, Veldhoven PJ et al. Record performance of a CW metallic subwavelength-cavity laser at room temperature. In: CLEO: science and innovations. OSA Technical Digest (online). Washington, DC: Optical Society of America, 2012: Paper CThM4.3.

41 Mizrahi A, Lomakin V, Slutsky BA, Nezhad MP, Feng L et al. Low threshold gain metal coated laser nanoresonators. Opt Lett 2008; 33: 1261-1263.

42 Rana F, Manolatou C. Subwavelength nanopatch cavities for semiconductor plasmon lasers. IEEE J Quantum Electron 2008; 44: 435-447.

43 Oulton RF, Sorger VJ, Genov DA, Pile DF, Zhang X. A hybrid plasmonic waveguide for subwavelength confinement and long-range propagation. Nat Photonics 2008; 2: 496-500.

44 Huang JQ, Kim SH, Scherer A. Design of a surface-emitting subwavelength metal-clad disk laser in the visible spectrum. Opt Express 2010; 18: 19581-19591.

45 Chang SW, Lin TR, Chuang SL. Theory of plasmonic Fabry-Perot nanolasers. Opt Express 2010; 18: 15039-15053.

46 Altug H, Englund D, Vuckovic J. Ultrafast photonics crystal nanocavity laser. Nat Phys 2006; 2 : 485-488.

47 Shore KA. Modulation bandwidth of metal-clad semiconductor nanolasers with cavityenhanced spontaneous emission. Electron Lett 2010; 46: 1688-1689.

48 MacDonald KF, Samson ZL, Stockman MI, Zheludev NI. Ultrafast active plasmonics. Nat Photonics 2009; 3: 55-58.

49 Marell MJ. Gap plasmon mode distributed feedback lasers. PhD dissertation, Technische Universiteit Eindhoven, Eindhoven, The Netherlands, 2011.

50 Chen R, Tran TT, Ng KW, Ko WS, Chuang LC et al. Nanolaser grown on silicon. Nat Photonics 2011; 5: 170-175.

51 VJ L, Kobayashi NP, Islam MS, Wu W, Chaturvedi P et al. Ultrasmooth silver thin films deposited with a germanium nucleation layer. Nano Lett 2009; 9: 178-182.

52 Chen WQ, Thoreson MD, Ishii S, Kildishev AV, Shalaev VM. Ultra-thin ultra-smooth and low-loss silver films on a germanium wetting layer. Opt Express 2010; 18: 5124 5134.

53 Kariniemi M, Niinistö J, Hatanpää T, Kemell M, Sajavaara T et al. Plasma-enhanced atomic layer deposition of silver thin films. Chem Mater 2011; 23: 2901-2907.

54 Fang $\mathrm{N}$, Lee $\mathrm{H}$, Sun $\mathrm{C}$, Zhang X. Sub-diffraction-limited optical imaging with a silver superlens. Science 2005; 308: 534-537.

$55 \mathrm{Li} \mathrm{D}$, Ning CZ. All-semiconductor active plasmonic system in mid-infrared wavelengths. Opt Express 2011; 19: 14594-14603.

56 Hoffman AJ, Alekseyev L, Howard SS, Franz KJ, Wasserman D et al. Negative refraction in semiconductor metamaterials. Nat Mater 2007; 6: 946-950.

57 Fang AW, Park H, Cohen O, Jones R, Paniccia MJ et al. Electrically pumped hybrid AIGalnAs-silicon evanescent laser. Opt Express 2006; 14: 9203-9210.

58 Vlasov Y, TJ Watson Research Center. 2008 ECOC Tutorial: On-Chip Si Nanophotonics, Silicon photonics for next generation computing systems. Yorktown Heights, NY: TJ Watson Research Center. 10 April 2011. Available at: http:// researcher.ibm.com/researcher/view_project.php?id=2757.

59 Kim MK, Lakhani AM, Wu MC. Efficient waveguide-coupling of metal-clad nanolaser cavities. Opt Express 2011; 19: 23504-23512.

60 Ding Q, Mizrahi A, Fainman Y, Lomakin V. Dielectric shielded nanoscale patch laser resonators. Opt Lett 2011; 36: 1812-1814.

(c) This work is licensed under a Creative Commons SOMEFाGHSHESEFVED Attribution-NonCommercial-NoDerivative Works 3.0 Unported License. To view a copy of this license, visit http:// creativecommons.org/licenses/by-nc-nd/3.0 